\title{
Predictors of outcomes following reablement in community-dwelling older adults
}

\author{
This article was published in the following Dove Press journal: \\ Clinical Interventions in Aging \\ 29 December 2016 \\ Number of times this article has been viewed
}

\author{
Hanne Tuntland ${ }^{1,2}$ \\ Ingvild Kjeken ${ }^{3,4}$ \\ Eva Langeland ${ }^{2,5}$ \\ Bjarte Folkestad ${ }^{2,6}$ \\ Birgitte Espehaug ${ }^{7}$ \\ Oddvar Førland ${ }^{2,8}$ \\ Mona Kristin Aaslund \\ 'Department of Global Public Health \\ and Primary Care, Faculty of Medicine \\ and Dentistry, University of Bergen, \\ ${ }^{2}$ Centre for Care Research Western \\ Norway, Bergen University College, \\ Bergen, ${ }^{3}$ National Advisory Unit \\ on Rehabilitation in Rheumatology, \\ Diakonhjemmet Hospital, \\ ${ }^{4}$ Department of Occupational \\ Therapy, Prosthetics and Orthotics, \\ Faculty of Health Sciences, Oslo \\ and Akershus University College of \\ Applied Sciences, Oslo, ${ }^{5}$ Department \\ of Nursing, Faculty of Health and \\ Social Sciences, Bergen University \\ College, ${ }^{6}$ Uni Research Rokkan \\ Centre, ${ }^{7}$ Centre for Evidence- \\ Based Practice, Bergen University \\ College, ${ }^{8}$ Faculty of Health Studies, \\ VID Specialized University, Campus \\ Bergen, Bergen, Norway
}

Background: Reablement is a rehabilitation intervention for community-dwelling older adults, which has recently been implemented in several countries. Its purpose is to improve functional ability in daily occupations (everyday activities) perceived as important by the older person. Performance and satisfaction with performance in everyday life are the major outcomes of reablement. However, the evidence base concerning which factors predict better outcomes and who receives the greatest benefit in reablement is lacking.

Objective: The objective of this study was to determine the potential factors that predict occupational performance and satisfaction with that performance at 10 weeks follow-up.

Methods: The sample in this study was derived from a nationwide clinical controlled trial evaluating the effects of reablement in Norway and consisted of 712 participants living in 34 municipalities. Multiple linear regression was used to investigate possible predictors of occupational performance (COPM-P) and satisfaction with that performance (COPM-S) at 10 weeks follow-up based on the Canadian Occupational Performance Measure (COPM).

Results: The results indicate that the factors that significantly predicted better COPM-P and COPM-S outcomes at 10 weeks follow-up were higher baseline scores of COPM-P and COPM-S respectively, female sex, having a fracture as the major health condition and high motivation for rehabilitation. Conversely, the factors that significantly predicted poorer COPM-P and COPM-S outcomes were having a neurological disease other than stroke, having dizziness/ balance problems as the major health condition and having pain/discomfort. In addition, having anxiety/depression was a predictor of poorer COPM-P outcomes. The two regression models explained $38.3 \%$ and $38.8 \%$ of the total variance of the dependent variables of occupational performance and satisfaction with that performance, respectively.

Conclusion: The results indicate that diagnosis, functional level, sex and motivation are significant predictors of outcomes following reablement.

Keywords: home-based rehabilitation, Canadian Occupational Performance Measure, aged, sex, frailty

\section{Introduction}

Reablement - or restorative care, as it is called in some countries - involves focused, time-limited interventions delivered in people's homes or outdoors in the community. It has been seen as a solution to a number of long-standing challenges in health care, including the cost pressures associated with a rapidly aging population, and is therefore being implemented in a growing number of countries. ${ }^{1}$ There is limited evidence, although not conclusive, that reablement leads to improved function in daily occupations, physical function and health-related quality of life for homedwelling older adults ${ }^{2-5}$ and to reduced costs and decreased demand for public health

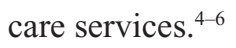

Correspondence: Hanne Tuntland Centre for Care Research Western Norway, Bergen University College, PO Box 7030, 5020 Bergen, Norway Tel +475558 7839

Email hanne.kristin.tuntland@hib.no 
Reablement focuses on supporting people to relearn skills and regain confidence in daily occupations. The rehabilitation model is goal-orientated, holistic and person-centered, designed to achieve goals that matter to each individual. ${ }^{1,5}$ The approach is multidisciplinary in nature and aims to help home-dwelling older people live independently and in fulfilling lives following functional decline, while simultaneously reducing the need for continuing support and long-term services. ${ }^{2}$ Reablement is an inclusive approach and seeks to work with all people who could benefit from this kind of support, irrespective of their age and diagnosis. ${ }^{1}$

In Norway, rehabilitation is a statutory service in primary care. Reablement is one form of rehabilitation that has rapidly spread across the country during the last 4.5 years. To date, more than one-third of Norwegian municipalities have implemented reablement, ${ }^{7}$ and the majority are using the Canadian Occupational Performance Measure (COPM) as the main instrument for goal determination and evaluation.

Despite an emerging evidence base on reablement internationally, very little is known about how the intervention is configured, the optimal timing and intensity and who receives the greatest benefit. ${ }^{1,3,8}$ Moreover, there is limited evidence on which elements are critical in determining its effectiveness and how the effectiveness may depend upon the characteristics of the participants. ${ }^{5,9}$ Consequently, there is a lack of knowledge concerning predictors of changed outcomes following reablement.

Hence, the objective of this study was to determine the potential factors that predict occupational performance and satisfaction with that performance at 10 weeks follow-up.

\section{Methods}

\section{Design and participants}

This is a prospective cohort study with a sample derived from a nationwide, clinical controlled trial aimed at evaluating the effects of reablement. ${ }^{4,10}$ The Norwegian Directorate of Health, which commissioned the study, granted municipalities financial support if attending the research project. Since the Norwegian Directorate of Health wanted adequate geographic representation and variability with regard to the amount of participants, measures were taken to ensure that municipalities from all parts of Norway and of various sizes were included. The whole sample consisted of 833 participants, of whom 712 people were in the intervention group receiving reablement and 121 people were in the control group receiving standard health care services. Data were collected at baseline, at 10 weeks follow-up and again after 6 and 12 months. A central allocation office in each municipality recruited consecutive participants to the study. The inclusion period lasted from April 1, 2014, until June 20, 2015, while the data collection ended on December 31, 2015. People were eligible if they were home-dwelling, $>18$ years of age, understood Norwegian and had experienced a functional decline. Participants were excluded if they were in need of institution-based rehabilitation or nursing home placement or if they were terminally ill or cognitively reduced (subjectively assessed by health care providers based on observation and communication). More details concerning the design of the study are available in the published protocol. ${ }^{10}$ An analysis comparing the participants who completed with the participants who dropped out in the clinical controlled trial showed no significant differences in baseline COPM-P and COPM-S scores $\left(P=0.87\right.$ and $P=0.83$, respectively) ${ }^{4}$

In this study, we included only the intervention group, as we wanted to examine the predictors of outcomes following reablement. Since having a large sample was important, we chose not to use data from the 6-month or 12-month data collection, as there would have been a high number of noncompleters and more people who dropped out. Besides, we wanted the results to be based on changes in outcomes after the intensive rehabilitation phase, not based on the subsequent follow-up periods. Consequently, our sample consisted of 712 participants living in 34 municipalities.

All participants received information about the study and gave written consent. The Regional Committee for Medical and Health Research Ethics for Western Norway approved the trial (REK West, 2014/57-1). The trial was registered at ClinicalTrials.gov on October 24, 2014, identifier: NCT02273934.

\section{Intervention fidelity}

As reablement was implemented in multiple municipalities, it was essential to train data collectors to ensure adherence to the protocol in all teams. A 1-day course was held on the use of the primary instrument, the COPM, where all municipalities sent one representative. We arranged another 1-day course for the contact persons in all municipalities focusing on the other instruments used and on the procedures of the research project with information on the required key elements of the reablement intervention. Hence, all data collectors were trained the same way. The contact person from each municipality also received a training manual with all the necessary information. The contact persons were given the responsibility of providing the requisite training to their respective team members. In addition, the project leader had close contact with all municipalities in the course of the 
data collection and implementation period in order to ensure intervention fidelity and follow-up of missing data. The reablement intervention consists of individual and general features as described elsewhere. ${ }^{10}$

\section{Data collection - dependent variables}

Occupational performance (COPM-P) and satisfaction with that performance (COPM-S), measured by the COPM, are typically used as primary outcomes in reablement research. ${ }^{4,6,11}$ Therefore, the COPM scores at 10 weeks follow-up were used as dependent variables in this study. The COPM instrument measures a person's self-perception of COPM-P and COPM-S outcomes in three areas: selfcare, productivity and leisure. ${ }^{12}$ During a semi-structured interview, participants described which occupations they experienced as important, but found difficult to perform. The term "occupation" is, in short, everything people do to occupy themselves, including looking after themselves, enjoying life, and contributing socially and economically to their communities. ${ }^{13}$ The importance of each occupation is rated on a $1-10$ point scale $(10=$ very important $)$. Next, the participant is asked to prioritize a maximum of five of the most important occupations and thereafter rate performance and satisfaction with performance for each of these occupations on a scale from 1 to 10 (higher score reflects better performance and higher satisfaction). Sum scores for the COPM-P and COPM-S, respectively, are calculated by adding the performance or satisfaction scores and thereafter dividing by the number of prioritized occupations. The COPM is found to have adequate psychometric properties in a home-dwelling older population. ${ }^{14}$

\section{Data collection - predictor variables}

We collected socio-demographic data from the participants. Age, sex, living status, educational level, motivation for rehabilitation and number of additional health conditions were used as independent variables, as well as ten categories of major health conditions. We used three different instruments to gather individual functional data at baseline. In addition, a questionnaire was sent to the contact person in each municipality, with questions about municipalityspecific details.

The first instrument used was the COPM, which measures occupational performance and satisfaction with that performance, an instrument that was described earlier. Baseline scores of COPM-P and COPM-S were used as independent variables. These outcomes were regarded as a measure of functional level.
The second instrument used was the Short Physical Performance Battery (SPPB), which is a physical test for balance, walking and muscle strength in the lower extremities. ${ }^{15}$ Based on the $4 \mathrm{~m}$ static start walking test included in the SPPB, preferred walking speed (PWS) was calculated and used as an independent variable. A walking speed $>1.0 \mathrm{~m} / \mathrm{s}$ is perceived as normal, a speed between 0.6 and $1.0 \mathrm{~m} / \mathrm{s}$ is perceived as initial disability and a walking speed $<0.6 \mathrm{~m} / \mathrm{s}$ is perceived as reflecting frailty. ${ }^{16}$ Good validity, reliability and responsiveness of the SPPB were shown in a systematic review involving studies where community-dwelling older adults were investigated. ${ }^{17}$

The third instrument used was the European Quality of Life Five Dimension Five Level Scale (EQ-5D-5L) that measures health-related quality of life. ${ }^{18}$ The instrument consists of a questionnaire and a visual analog scale (VAS). The questionnaire has five domains (mobility, self-care, usual activities, pain/discomfort and anxiety/depression) with five levels ( $1=$ no problems, $5=$ extreme problems). The health status today VAS score gives an indication of how the participants assess their own health on a $0-100$ scale, with 100 being excellent health. The domains of pain/discomfort, anxiety/ depression and health status today were used as independent variables. A structured review of the psychometric properties of the EQ-5D-5L concluded that there is good evidence for reliability, validity and responsiveness among older adults. ${ }^{19}$

Finally, one question about the organizational team model from the municipal questionnaire was used. The organizational model within the home-based services comprises three different categories. The first and most common team model is a home-care services integrated model, where the nurses, auxiliary nurses and assistants work closely together with the occupational therapist and physiotherapist (the home-based rehabilitation services). In the second model, the rehabilitation services integrated model, the reablement team is located in the rehabilitation services in collaboration with the home-care services. The third model is a specialist team model, comprising a multidisciplinary team that works only with reablement tasks and covers a larger district. Except for the three organizational models, all independent variables are presented in Table 1.

\section{Data analysis}

Continuous variables were described by means and standard deviations (SD), whereas categorical variables were described by frequencies and percentages.

Linear regression analyses were performed to investigate associations between COPM-P and COPM-S measured at 
Table I Participant characteristics at baseline

\begin{tabular}{|c|c|}
\hline Variables & Total $(\mathbf{N}=7 \mid 2)$ \\
\hline Age, mean (SD), min-max, years & 78.2 (11.2), 19-95 \\
\hline Sex, females, frequency (\%) & $487(68.7)$ \\
\hline Living alone, frequency yes (\%) & $50 \mathrm{I}(70.6)$ \\
\hline Higher education, frequency yes (\%) & $140(16.7)$ \\
\hline \multicolumn{2}{|l|}{ Major health condition, frequency (\%) } \\
\hline Fractures & 147 (20.7) \\
\hline Dizziness/balance problems & $113(15.9)$ \\
\hline Orthopedic disease & $73(10.3)$ \\
\hline Pain & $70(9.8)$ \\
\hline Stroke & $67(9.4)$ \\
\hline Arthritis & $47(6.6)$ \\
\hline Heart disease & $37(5.2)$ \\
\hline Pulmonary disease & $29(4.1)$ \\
\hline Neurological disease other than stroke & $24(3.4)$ \\
\hline Other health condition & $100(14.0)$ \\
\hline $\begin{array}{l}\text { Number of additional health conditions, } \\
\text { mean (SD), min-max }\end{array}$ & $2.28(2.02), 0-10$ \\
\hline $\begin{array}{l}\text { Motivation for rehabilitation, scale } 1-10 \text {, } \\
10 \text { is best, mean (SD), min-max }\end{array}$ & $8.2(2.0), 1-10$ \\
\hline $\begin{array}{l}\text { Occupational performance, COPM-P, scale } \\
I-10,10 \text { is best, mean (SD), min-max }\end{array}$ & $3.4(1.6), 1.0-8.5$ \\
\hline $\begin{array}{l}\text { Occupational satisfaction, COPM-S, scale } \\
1-10,10 \text { is best, mean (SD), min-max }\end{array}$ & $3.4(1.8), 1.0-8.6$ \\
\hline PWS test, $\mathrm{m} / \mathrm{s}$, mean (SD), min-max & $0.5(0.2), 0.1-1.9$ \\
\hline $\begin{array}{l}\text { Health status today, EQ VAS, scale } 0-100 \text {, } \\
100 \text { is best, mean (SD), min-max }\end{array}$ & $48.6(19.3), 0-100$ \\
\hline $\begin{array}{l}\text { Anxiety/depression, EQ-5D-5L, scale } I-5 \text {, } \\
I \text { is best, mean (SD), min-max }\end{array}$ & $1.9(0.9), I-5$ \\
\hline $\begin{array}{l}\text { Pain/discomfort, EQ-5D-5L, scale } I-5, I \text { is } \\
\text { best, mean (SD), min-max }\end{array}$ & $2.7(1.0), 1-5$ \\
\hline
\end{tabular}

Abbreviations: COPM, Canadian Occupational Performance Measure; COPM-P, COPM measuring occupational performance; COPM-S, COPM measuring satisfaction with performance; EQ-5D-5L, European Quality of Life Five Dimension Five Level Scale; min, minimum; max, maximum; SD, standard deviation; VAS, visual analog scale.

10 weeks and a selected set of baseline variables. Univariate analyses were first performed to screen for predictor variables for COPM-P and COPM-S. The choice of independent variables used in the univariate analyses was based on the review of literature and clinical judgment. In the next step, variables that were statistically significant at a $P<0.2$ level in the univariate analyses were included in multivariate regression models with additional adjustment for baseline levels of COPM-P and COPM-S, respectively.

Estimated regression coefficients from the univariate and multivariate regression analyses were reported with $95 \%$ confidence intervals and $P$-values. The $R^{2}$ (coefficient of determination) and the root mean square error (RMSE) were reported as goodness-of-fit indicators of the regression models.

Regression diagnostics were performed to investigate any violation of the assumptions of normality, linearity, multicollinearity and homoscedasticity.
Secondary analyses were performed with interaction terms included in the regression models to assess whether an association between fracture and COPM was dependent on sex.

$P$-values $<0.05$ were considered statistically significant. Statistical analyses were performed with IBM SPSS Statistics version 23 (IBM Corporation, Armonk, NY, USA).

\section{Results}

The main baseline socio-demographic and functional characteristics are presented in Table 1. The participants were mostly female, had no higher education and were living alone. The mean age was 78 years. Fractures and dizziness/balance problems were the most frequent reasons for needing rehabilitation. The functional characteristics measured not only by PWS but also by COPM and EQ-5D VAS scores demonstrated that the participants had moderate to severe disability. Approximately $15 \%$ of the Norwegian population was living in the municipalities included in this study. The municipalities geographically stretched from the south to the north of Norway. The dropout rates for COPM-P and COPM-S were $17.1 \%$ and $17.4 \%$, respectively, at 10 weeks follow-up.

The results from the univariate regression analyses of the associations between baseline variables and the COPM-P score at 10 weeks follow-up are presented in Table 2. All independent variables with $P<0.2$ are presented in Table 2 . Factors with higher $P$-values were PWS, number of additional health conditions and home-care services integrated model versus specialist team model.

Table 2 also displays the results of the multivariate regression analysis for COPM-P (Model 1). Higher baseline scores for COPM-P were associated with better COPM-P scores $(b=0.20, P=0.001)$. Moreover, having a fracture as the major health condition predicted better outcomes $(b=0.73$, $P=0.001)$. In addition, female sex and high motivation were significant predictors of higher COPM-P scores after 10 weeks. The home-care services integrated model versus the rehabilitation services integrated model did not reach statistical significance $(P=0.054)$.

Having a neurological disease other than stroke and having dizziness/balance problems as the major health condition significantly predicted poorer COPM-P outcomes. This was also observed for having pain/discomfort and having anxiety/ depression. The model explained $38.3 \%$ of the total variance of the dependent variable COPM-P. The RMSE was equal to 1.93 .

The results from the univariate regression analyses of the associations between baseline variables and the COPM-S score at 10 weeks follow-up are presented in Table 3. All 
Table 2 Factors at baseline associated with COPM-P outcomes at 10 weeks follow-up (Model I)

\begin{tabular}{|c|c|c|c|c|c|c|}
\hline \multirow[t]{2}{*}{ Variables } & \multicolumn{3}{|c|}{ Univariate regression $(\mathrm{N}=\mathbf{5 9 0})$} & \multicolumn{3}{|c|}{ Multivariate regression $(\mathrm{N}=590)$} \\
\hline & $b$ & $95 \% \mathrm{Cl}$ & $P$-value & b & $95 \% \mathrm{Cl}$ & $P$-value \\
\hline Age & 0.01 & $-0.09,0.03$ & 0.12 & 0.01 & $-0.02,0.17$ & 0.94 \\
\hline Sex (female) & 0.53 & $0.17,0.89$ & 0.004 & 0.49 & $0.13,0.85$ & 0.008 \\
\hline Fracture (as major health condition) & 1.10 & $0.70,1.50$ & $<0.001$ & 0.73 & $0.31,1.15$ & 0.001 \\
\hline Dizziness/balance problems (as major health condition) & -0.58 & $-1.04,-0.11$ & 0.02 & -0.79 & $-1.26,-0.32$ & 0.001 \\
\hline Neurological disease (as major health condition) & -1.17 & $-2.13,-0.22$ & 0.02 & -1.29 & $-2.3 \mathrm{I},-0.28$ & 0.01 \\
\hline Motivation for rehabilitation, scale $1-10,10$ is best & 0.16 & $0.07,0.24$ & $<0.001$ & 0.13 & $0.05,0.22$ & 0.003 \\
\hline Occupational performance, COPM-P, scale $I-10,10$ is best & 0.18 & $0.07,0.29$ & 0.001 & 0.20 & $0.08,0.31$ & 0.001 \\
\hline Occupational satisfaction, COPM-S, scale $I-10,10$ is best & 0.17 & $0.07,0.26$ & 0.001 & - & - & - \\
\hline Pain/discomfort, EQ-5D-5L, scale $I-5, I$ is best & -0.25 & $-0.4 I,-0.08$ & 0.003 & -0.19 & $-0.37,-0.02$ & 0.03 \\
\hline Anxiety/depression, EQ-5D-5L, scale $I-5, I$ is best & -0.25 & $-0.43,0.06$ & 0.009 & -0.19 & $-0.38,-0.00$ & 0.05 \\
\hline Health status today, EQ VAS, scale $0-100,100$ is best & 0.02 & $0.01,0.03$ & $<0.001$ & 0.01 & $-0.00,0.15$ & 0.28 \\
\hline $\begin{array}{l}\text { Home-care services integrated model versus rehabilitation } \\
\text { services integrated } \text { model }^{a}\end{array}$ & -0.34 & $-0.74,-0.05$ & 0.09 & -0.37 & $-0.75,0.01$ & 0.05 \\
\hline
\end{tabular}

Notes: $b$ denotes unstandardized coefficients. ${ }^{a}$ Favors home-care services integrated model.

Abbreviations: $\mathrm{Cl}$, confidence interval; COPM, Canadian Occupational Performance Measure; COPM-P, COPM measuring occupational performance; COPM-S, COPM measuring satisfaction with performance; EQ-5D-5L, European Quality of Life Five Dimension Five Level Scale; VAS, visual analog scale.

independent variables with $P<0.2$ are displayed in Table 3. Factors with higher $P$-values were PWS, home-care services integrated team model versus specialist team model and home-care services integrated team model versus rehabilitation services integrated model.

Table 3 also shows the results of the multivariate regression analysis for COPM-S (Model 2). Higher baseline scores for COPM-S were associated with better COPM-S scores at 10 weeks follow-up $(b=0.23, P \leq 0.001)$. Furthermore, being female predicted better outcomes ( $b=0.63, P=0.002$ ). In addition, having a fracture as the major health condition and high motivation were significant predictors of higher COPM-S scores after 10 weeks.
Having dizziness/balance problems as the major health condition and having a neurological disease other than stroke were significant predictors of poorer COPM-S outcomes. In addition, having pain/discomfort significantly predicted poorer outcomes. The model explained $38.8 \%$ of the total variance of the dependent variable COPM-S. The RMSE was equal to 2.07 .

Analyses showed no violation of the assumptions of normality, linearity, multicollinearity and homoscedasticity in any of the models, although there was a high correlation between baseline scores of COPM-P and COPM-S $(r=0.75$, $P=0.01)$. These factors were therefore not entered into the same regression models.

Table 3 Factors at baseline associated with better COPM-S outcomes at 10 weeks follow-up (Model 2)

\begin{tabular}{|c|c|c|c|c|c|c|}
\hline \multirow[t]{2}{*}{ Variable } & \multicolumn{3}{|c|}{ Univariate regression $(\mathbf{N}=\mathbf{5 8 8})$} & \multicolumn{3}{|c|}{ Multivariate regression $(\mathrm{N}=\mathbf{5 8 8})$} \\
\hline & $b$ & $95 \% \mathrm{Cl}$ & $P$-value & $b$ & $95 \% \mathrm{Cl}$ & P-value \\
\hline Age & 0.02 & $0.00,0.04$ & 0.02 & 0.01 & $-0.01,0.02$ & 0.55 \\
\hline Sex (female) & 0.64 & $0.25,1.02$ & 0.001 & 0.63 & $0.24,1.01$ & 0.002 \\
\hline Fracture as major health condition & I.0I & $0.59,1.44$ & $<0.001$ & 0.58 & $0.13,1.02$ & 0.01 \\
\hline Dizziness/balance problems as major health condition & -0.62 & $-1.11,-0.12$ & 0.02 & -0.85 & $-1.35,-0.35$ & 0.001 \\
\hline Neurological disease as major health condition & -1.65 & $-2.66,-0.64$ & 0.001 & -1.75 & $-2.82,-0.67$ & 0.02 \\
\hline Number of additional health conditions & -0.09 & $-0.18,-0.00$ & 0.04 & -0.02 & $-0.1 \mathrm{I}, 0.07$ & 0.68 \\
\hline Motivation for rehabilitation, scale $1-10,10$ is best & 0.11 & $0.02,0.20$ & 0.02 & 0.11 & $0.01,0.20$ & 0.03 \\
\hline Occupational performance, COPM-P, scale $1-10,10$ is best & 0.14 & $0.03,0.26$ & 0.02 & - & - & - \\
\hline Occupational satisfaction, COPM-S, scale $1-10,10$ is best & 0.24 & $0.14,0.35$ & $<0.001$ & 0.23 & $0.12,0.34$ & $<0.001$ \\
\hline Pain/discomfort, EQ-5D-5L, scale $\mathrm{I}-5, \mathrm{I}$ is best & -0.30 & $-0.47,-0.13$ & 0.001 & -0.24 & $-0.42,-0.06$ & 0.01 \\
\hline Anxiety/depression, EQ-5D-5L, scale $1-5, I$ is best & -0.27 & $-0.46,-0.07$ & 0.007 & -0.17 & $-0.38,0.03$ & 0.09 \\
\hline Health status today, EQ VAS, scale $0-100,100$ is best & 0.02 & $0.01,0.03$ & $<0.001$ & 0.00 & $-0.01,0.01$ & 0.51 \\
\hline
\end{tabular}

Note: $b$ denotes unstandardized coefficients.

Abbreviations: $\mathrm{Cl}$, confidence interval; COPM, Canadian Occupational Performance Measure; COPM-P, COPM measuring occupational performance; COPM-S, COPM measuring satisfaction with performance; EQ-5D-5L, European Quality of Life Five Dimension Five Level Scale; VAS, visual analog scale. 
Secondary analyses showed that sex was not a statistically significant moderator of the association between COPM-P ( $P=0.93)$ and COPM-S $(P=0.36)$ outcomes, respectively, and having a fracture.

\section{Discussion}

To the best of our knowledge, this is the first study identifying predictors of outcomes following reablement. The results demonstrate that a high baseline COPM score, female sex, having a fracture as the major health condition and high motivation for rehabilitation significantly predict both better occupational performance and higher satisfaction with performance 10 weeks after starting reablement. Conversely, the predictors of poorer performance and satisfaction with performance after 10 weeks are having a neurological disease other than stroke, having dizziness/balance problems as the major health condition and having high levels of pain/discomfort. In addition, having anxiety/depression is a predictor of poorer occupational performance.

Having dizziness/balance problems as the major health condition was a significant predictor for poorer performance and lower satisfaction with performance after 10 weeks. An explanation for this finding may be that dizziness and balance problems lead to fear of falling and limited activity. ${ }^{20}$ Many older people live alone and feel more secure when professionals are present in their homes and guide them when performing daily occupations. ${ }^{21}$ Older people with dizziness and balance problems may not dare to practice on their own in daily occupations involving mobility, and, therefore, they show less progress.

A neurological disease other than stroke was also a predictor of poorer COPM outcomes. One reason for this may be that many neurological diseases are chronic and also progressive in nature with fewer prospects of improvement. This category is a collective term, and the results do not reveal to which neurological diagnoses the predictor of poorer outcomes apply. Hence, we have no knowledge of which neurological diagnoses are advantageous or disadvantageous in this respect. Future research should conduct subgroup analysis in order to determine this.

Moreover, having anxiety/depression and pain/discomfort are predictors of poorer outcomes. Chronic pain affects people's well-being and the ability to maintain an independent and active life. Mood and anxiety disorders have been found to be associated with chronic pain. ${ }^{22}$ Hence, the explanation for why these health conditions predict poorer outcomes may be that the health conditions by their nature are conditions that represent increased immobility and passivity, which implies that progress in reablement is dependent on active engagement and intensive practice.

Having a fracture diagnosis, on the other hand, is a strong predictor of better functional outcomes. Another study of people with a hip fracture supports this finding. ${ }^{23}$ This is despite the fact that fracture is also a collective term encompassing various types of fractures with different prospects of recovery. An explanation for this finding may be that a fracture is an acute traumatic event, and improvement is expected as the fracture heals during the first weeks and months after the injury.

In summary, an important finding in this study is that diagnosis matters in reablement. In this study, several diagnoses or health conditions were found to be predictors of better or poorer outcomes. Hence, the results show that the outcomes of reablement are not irrespective of diagnosis as previously assumed. ${ }^{1}$ Due to the general features of the intervention, reablement may in some respects be considered as "one size fits all" intervention. Anyway, a "one size fits all" intervention is unlikely to suit most participants. ${ }^{24}$ However, as this is not a controlled study, there is, based on our research, insufficient evidence to support a shift of target group, for instance omitting certain diagnoses. We do not know if the participants' function would have deteriorated more without the reablement intervention. However, individual adjustments according to diagnosis may be needed in order to optimize the intervention.

Higher baseline scores were found to be associated with better COPM outcomes at 10 weeks follow-up. We find it reasonable that the participants, who scored highest initially, did the same at follow-up. Likewise, the ones who scored lowest initially also scored lowest at follow-up. Hence, the results are a reflection of the participants' development during the rehabilitation phase. Since we did not examine the change scores from baseline to follow-up, the results do not reveal which of the two groups improved the most. Therefore, the results only imply that there is an association between the pre-assessment and post-assessment functional level with regard to COPM outcomes.

A novel finding in this study is that female sex was a predictor of better COPM outcomes. Sex differences have been found in many health-related aspects, for instance in longevity in old age. ${ }^{25}$ There are also sex differences in morbidity among older people. ${ }^{26}$ Therefore, we had a hypothesis that female sex was a moderator of the association between COPM outcomes and having a fracture, but this hypothesis was not confirmed. Hence, we do not know why women benefit more from reablement than men do. 
As could be expected, high motivation is found to be a predictor of better COPM outcomes. Motivation for rehabilitation may be present before the start of reablement or be developed during the COPM interview or in the rehabilitation phase. The importance of motivation in reablement is supported by a qualitative study, where the dynamic interactions between intrinsic and extrinsic motivational factors were found to be the main driving forces. ${ }^{21}$ Intrinsic motivation was based on the person's own willpower and responsibility, whereas extrinsic motivation was enhanced in cooperation with the reablement team. This dynamic interaction is also believed to occur during the COPM interview. The interview allows participants to identify problems in their self-care, productivity and leisure occupations and prioritize the most important of these. Hence, the participants' intrinsic motivation may be stimulated through increased awareness when defining their own goals, and their extrinsic motivation may be enhanced by the professional staff's support and engagement. ${ }^{21}$ It is also recognized that goals defined by the participants themselves motivate the most. ${ }^{27}$ In the literature, clear personalized goals for the outcomes of reablement have been found to promote motivation. ${ }^{28}$ Moreover, it has been found that using a patient-specific instrument such as COPM enhances participation ${ }^{29}$ and results, ${ }^{30}$ while a staffdirected goal-setting and rehabilitation process reduces participant engagement. ${ }^{31}$

Even if the inclusion criterion regarding age was 18 years or older, the recruitment gave a sample of advanced age with a mean age of 78 years. Therefore, with the main proportion of the sample being old, it was not possible to provide results indicating a significant association between age and COPM outcomes. However, the results do imply that reablement can be offered to people of all ages, as stated also elsewhere. ${ }^{1}$

The results indicated a tendency where a reablement team that derives from a municipal home-based service integrated team model in cooperation with rehabilitation services seems to provide better COPM-P outcomes, although these results were not statistically significant. This organizational model means that nurses, auxiliary nurses and assistants are the basis of the team in collaboration with rehabilitation services. Successful reablement requires an approach that stimulates the participants to do the occupations themselves. ${ }^{2}$ Our results indicate that teams from the home-based services give better care-staff commitment for this approach and therefore a tendency for better outcomes after 10 weeks.

The total variance of the dependent variables explained by the two models was 38\%-39\% indicating that factors not explored in this study exist that can further explain COPM outcomes after reablement. The professionals' competence and the quality of their relationship with the participants may be factors that can have an impact. Future research may explore these possible explanations. We found that outcomes of COPM-P and COPM-S are predicted by nearly the same factors and the models have very similar explained variances, indicating that the two dependent variables are closely related. As a result, one may question if it is necessary to have these two dimensions in the same instrument. It may be sufficient to only measure the dimension of occupational performance (COPM-P), since the dimension satisfaction with performance (COPM-S) does not seem to contribute with additional information.

A major strength of this study is the large sample, which allowed multiple relevant variables to be tested. It has been suggested that for regression analysis, at least 20 participants are required for each factor studied. ${ }^{32}$ In this study, a maximum of 11 potential predictors were included in the regression analyses. With a sample of 588 participants at follow-up, we had enough power to assess this number of independent variables.

Another strength is the diagnostic and geographical heterogeneity among the participants, which implies that the results may be generalizable to the population of home-dwelling older adults with functional decline. There was also a great variety in health professionals providing the reablement intervention. Moreover, the study was conducted in a real-life context in multiple settings in primary care, which contributes to the representativeness of the results. However, a study consisting of 34 municipalities with multiple data collectors and health care providers presents a challenge to ensuring standardization of data collection procedures and the intervention delivered. Since measures were taken to ensure reliability in data collection and intervention fidelity, we found that the advantages surpassed the disadvantages in this respect.

A limitation in this study is that data from the control group were not included in the analyses. Hence, we cannot draw strong conclusions regarding the clinical implications of the results. However, we have pointed out some implications for future research.

Another limitation in this study is the moderate dropout at 10 weeks follow-up, which does represent a possibility of selection bias. We do not consider this as a general problem, since an analysis comparing the participants who completed and the participants who dropped out in the clinical controlled trial showed no significant differences in baseline COPM-P and COPM-S scores. However, we did not perform retention analyses in relation to diagnosis. Hence, we do not know 
whether there are systematic dropouts regarding specific diagnoses. In addition, the short time frame of 10 weeks is a limitation in this prediction study.

\section{Conclusion}

Reablement is not an intervention that is irrespective of diagnosis as previously assumed. As a result, individual adjustments according to diagnosis may be needed in order to optimize the intervention. The results indicate that diagnosis, functional level, sex and motivation at baseline are significant predictors of outcomes following reablement at 10 weeks follow-up.

\section{Acknowledgments}

We want to thank all the participants and the health care providers who participated in this trial. In addition, we want to thank the Norwegian Directorate of Health who commissioned the study.

\section{Disclosure}

The authors report no conflicts of interest in this work.

\section{References}

1. Aspinal F, Glasby J, Rostgaard T, Tuntland H, Westendorp RGJ. Reablement: supporting older people towards independence. Age Ageing. 2016;45(5):574-578.

2. Tessier A, Beaulieu M-D, MCGinn CA, Lautulippe R. Effectiveness of reablement: a systematic review. Healthc Policy. 2016;11(4):49-59.

3. Whitehead PJ, Worthington EJ, Parry RH, Walker MF, Drummond AE. Interventions to reduce dependency in personal activities of daily living in community dwelling adults who use homecare services: a systematic review. Clin Rehabil. 2015;29(11):1064-1076.

4. Langeland E, Førland O, Aas E, et al. Modeller for hverdagsrehabilitering - en følgeevaluering i norske kommuner. Effekter for brukerne og gevinster for kommunene? [Models of reablement. A study in Norwegian muncipalities. Effects for users and gains for municipalities?]. Available from: https://brage.bibsys.no/xmlui/bitstream/ handle/11250/2389813/1/Rapport6_16_web.pdf. Accessed November $10,2016$.

5. Cochrane A, McGilloway S, Furlong M, Molloy D, Stevenson M, Donnoly M. Time-limited home-care reablement for maintaining and inproving the functional independence of older adults (Review). Cochrane Database Syst Rev. 2016;10:CD010825.

6. Kjerstad E, Tuntland H. Reablement in community-dwelling older adults: a cost-effectiveness study analysis alongside a randomized controlled trial. Health Econ Rev. 2016;6(15:1-10).

7. Ness NE. Hverdagsrehabilitering-ingen grunn tilå vente [Reablement-no reason to wait]. Dagens Medisin [Medicine of Today]. 2016 Sept 6.

8. Ryburn B, Wells Y, Foreman P. Enabling independence: restorative approaches to home care provision for frail older adults. Health Soc Care Community. 2009;17(3):225-234.

9. Lewin G, Concanen K, Youens D. The Home Independence Program with non-health professionals as care managers: an evaluation. Clin Interv Aging. 2016;11:807-817.

10. Langeland E, Tuntland H, Førland O, et al. Study protocol for a multicenter study of reablement in Norway. BMC Geriatr. 2015;15(111):1-9.

11. Tuntland H, Aaslund M, Espehaug B, Førland O, Kjeken I. Reablement in community-dwelling older adults: a randomised controlled trial. $B M C$ Geriatr. 2015;15(146):1-11.
12. Law M, Baptiste S, Carswell A, McColl M, Polatajko H, Pollock N. COPM Canadian Occupational Performance Measure (Norwegian Version). 5 ed. Oslo: NKRR National Advisory Unit on Rehabilitation in Rheumatology; 2015.

13. Townsend E, Polatajko H. Enabling Occupation II. Advancing an Occupational Therapy Vision for Health, Well-Being and Justice through Occupation. Canada: Canadian Association of Occupational Therapists; 2007.

14. Tuntland H, Aaslund MK, Langeland E, Espehaug B, Kjeken I. Psychometric properties of the Canadian Occupational Performance Measure in home-dwelling older adults. J Multidiscip Healthc. 2016; 6(9):411-423.

15. Guralnik J, Simonsick E, Ferruci K, et al. A short physical performance battery assessing lower extremity function: association with self-reported disability and prediction of mortality and nursing home admission. J Gerontol. 1994;49(2):M85-M94.

16. Studenski S, Perera S, Wallace D, et al. Physical performance measures in the clinical setting. J Am Geriatr Soc. 2003;51(3):314-322.

17. Freiberger E, De Vreede P, Shoene D, et al. Performance-based physical function in older community-dwelling persons: a systematic review of instruments. Age Ageing. 2012;41(6):712-721.

18. Euroqol-Group. EuroQol - a facility for the measurement of healthrelated quality of life. Health Policy. 1990;16:199-208.

19. Haywood KL, Garratt AM, Fitzpatrick R. Quality of life in older people: a structured review of generic self-assessed health instruments. Qual Life Res. 2005;14(7):1651-1668.

20. Okubo Y, Osuka Y, Jung S, et al. Walking can be more effective than balance training in fall prevention among community-dwelling older adults. Geriatr Gerontol Int. 2016;16(1):118-125.

21. Hjelle KM, Tuntland H, Førland $\mathrm{O}$, Alvsvåg H. Driving-forces for home-based reablement: a qualitative study of older adults' experiences. Health Soc Care Commun. Epub 2016 Jan 24.

22. Tsang A, Von Korff M, Lee S, et al. Common chronic pain conditions in developed and developing countries: gender and age differences and comorbidity with depression-anxiety disorders. J Pain. 2008; 9(10):883-891.

23. Mizrahi EH, Arad M, Fleissig Y, Adunsky A. Gender differences in functional outcome of elderly hip fracture patients. Geriatr Gerontol Int. 2014;14(4):845-850.

24. Legg L, Gladman J, Drummond A, Davidson A. A systematic review of the evidence on home care reablement services. Clin Rehabil. 2016; 30(8):741-749.

25. World Health Organization. World Health Statistics 2014. Large Gains in Life Expectancy. Geneva: World Health Organization; 2014.

26. Luppa M, Luck T, Weyerer S, Konig HH, Riedel-Heller SG. Gender differences in predictors of nursing home placement in the elderly: a systematic review. Int Psychogeriatr. 2009;21(6):1015-1025.

27. Rostgaard T, Graff L. Med hoenderne i lommen. Borger og medarbejders samspil og samarbejde i rehabilitering [With Hands in the Pockets. Citizen and Employee Interaction and Cooperation in Rehabilitation]. Copenhagen: KORA. Danish Institute for Local and Regional Government Research; 2016.

28. Newton C. Personalising reablement: inserting missing link. Work Older People. 2012;16(3):117-121.

29. Wressle E, Eeg-Olofsson AM, Marcusson J, Henriksson C. Improved client participation in the rehabilitation process using a client-centred goal formulation structure. J Rehabil Med. 2002;34(1):5-11.

30. Parsons JGM, Parsons MJG. The effect of a designated tool on personcentred goal identification and service planning among older people receiving homecare in New Zealand. Health Soc Care Commun. 2012; 20(6):653-662.

31. Lexell E, Lexell J, Larsson-Lund M. The rehabilitation plan can support clients' active engagement and facilitate the process of change experiences from people with late effects of polio participating in a rehabilitation programme. Disabil Rehabil. 2016;38(4):329-336.

32. Polit D, Beck C. Nursing Research. Generating and Assessing Evidence for Nursing Practice. 10 ed. Philadelphia: Wolters Kluwer Health; 2017. 


\section{Publish your work in this journal}

Clinical Interventions in Aging is an international, peer-reviewed journal focusing on evidence-based reports on the value or lack thereof of treatments intended to prevent or delay the onset of maladaptive correlates of aging in human beings. This journal is indexed on PubMed Central, MedLine,
CAS, Scopus and the Elsevier Bibliographic databases. The manuscript management system is completely online and includes a very quick and fair peer-review system, which is all easy to use. Visit http://www.dovepress. $\mathrm{com} /$ testimonials.php to read real quotes from published authors. 